Trinity University

Digital Commons @ Trinity

Human Communication and Theatre Faculty

Research

Human Communication and Theatre

$9-5-2016$

\title{
The Relationship Between Instagram Selfies and Body Image in Young Adult Women
}

Charles N. Wagner

Trinity University, cwagner@trinity.edu

Ester Aguirre Alfaro

Trinity University, eaguirre@trinity.edu

Erin M. Bryant

Trinity University, ebryant@trinity.edu

Follow this and additional works at: https://digitalcommons.trinity.edu/hct_faculty

Part of the Theatre and Performance Studies Commons

\section{Repository Citation}

Wagner, C., Aguirre, E., \& Sumner, E. M. (2016). The relationship between Instagram selfies and body image in young adult women. First Monday, 21(9). doi: 10.5210/fm.v21i9.6390

This Article is brought to you for free and open access by the Human Communication and Theatre at Digital Commons@ Trinity. It has been accepted for inclusion in Human Communication and Theatre Faculty Research by an authorized administrator of Digital Commons @ Trinity. For more information, please contact jcostanz@trinity.edu. 


\title{
The relationship between Instagram selfies and body image in young adult women by Charles Wagner, Ester Aguirre, and Erin M. Sumner
}

\begin{abstract}
This study observed the relations between actual body size, body dissatisfaction, frequency of selfies taken, and number of Instagram selfies posted. Results indicated that actual body size was positively related to body dissatisfaction, and negatively related to the number of selfies taken. Results also revealed a positive relationship between body dissatisfaction and selfies taken. Conversely, no correlations were detected between the frequency of selfies posted to Instagram and either actual body size or body image dissatisfaction.
\end{abstract}

\section{Contents}

Introduction

Literature review

Methods

Results

Discussion

Limitations and conclusions

\section{Introduction}

The term millennial has gained popularity in reference to the age cohort that directly follows Generation-X (Howe and Strauss, 2000; Strauss and Howe, 1991). The Pew Research Center defines millennials as individuals born between 1981 and 1997 (Fry, 2016). In their body of work regarding generational theory, Strauss and Howe argue that each generation takes on unique traits and forms a sense of shared identity due to their shared social and historical experiences. Millennials are still establishing their collective adult identity, but are often characterized by their status as the first generation to have come of age in the digital era.

During the latter part of the twentieth century, most media socialization occurred in the form of one-to-many television and radio broadcasts designed for collective family viewing. The Internet has ushered a change in media habits, particularly for millennials who have never experienced life without digital technology. For example, the mass adoption of mobile media devices (e.g., tablets, cell phones, and laptops) has created a "bedroom culture" in which millennials partake in highly personalized and private media worlds (Livingstone, 2002). This shift in media is further compounded by the rise of social network sites (SNS) and other online venues that enable individuals to construct and display their identities while interacting with other network members (boyd and Ellison, 2007). The enlarged sense of media personalization and tendency to use the Internet for social purposes has led many millennials to become increasingly invested in developing an idealized online self that they can present to the world (Gonzales and Hancock, 2011). The pursuit of an idealized online self could, however, provide a dangerous playground for adolescents and young adults who suffer from body dissatisfaction or other esteem issues.

Instagram is an image-based SNS that is particularly popular among the millennial generation. Instagram allows users to take photos, post these photos to their personalized pages, and further disseminate the content by linking through other social media accounts. The popularity of Instagram is often linked with the rise of a new social phenomenon known as selfies (i.e., self-portrait style photographs). The selfie phenomenon has gained a great deal of public attention, with selfie being named the 2013 word of the year by Oxford Dictionaries [1]. Despite its social importance, the selfie phenomenon remains relatively untested within the scholarly community. This void is noteworthy because selfies might help Instagram users display their identities in a physical or body-centric manner. A selfie's focus on physical form might come with unintended associations for users who possess different levels of confidence and comfort with their bodies. The present study seeks to address this issue by examining the prevalence of selfie taking and posting among young women of the millennial generation, while seeking to 
determine whether the frequency of selfies taken and posted to Instagram are related to users' actual body size and sense of body dissatisfaction. This knowledge will help identify the extent to which the selfie trend is tied to larger issues of body image for young women.

\section{Literature review}

\section{Female body image}

Body image refers to the perceptions and attitudes that individuals hold about their own bodies in relation to larger cultural expectations (Davison and McCabe, 2005). As summarized by Szymanski, et al. (2011), body image involves selfassessments of many different bodily features such as skin tone, proportions, and size. Body image is important because it entails making social and cultural comparisons that might feed into a person's sense of physical attractiveness and larger selfworth (Cash, 2002). Although body image is important for both men and women, objectification theory (Fredrickson and Roberts, 1997) asserts that women are more likely to be seen as physical and sexual objects whose social value can be inferred from bodily appearance. As such, women are more likely to engage in forms of self-objectification that confirm the sense of connection between their physical bodies and their sense of self-worth.

Expectations regarding the ideal female body size and shape have varied across time and culture. In modern Western society the ideal female body typically involves forms of thinness that might range from toned and athletic to slim and lanky (Pelegrini, et al., 2014). The current privileging of the thin female body could possess important ramifications for millennial women. More than half of the United States adult population can be classified as either overweight or obese, which reflects a growing sense of discrepancy between real bodies and social norms of female body beauty (Pesa, et al., 2000). Indeed, approximately half of American girls aged 11-16 report being unhappy with their body image (White and Halliwell, 2010). Although women tend to report greater body dissatisfaction than men (Furnham, et al., 2002), existing research has revealed a correlation between body image and self-esteem among both young adult men (Olivardia, et al., 2004) and young adult women (Fabian and Thompson, 1989). Individuals who possess a poor sense of body image are also more likely to develop mental and physical issues such as eating disorders (Dittmar, 2009). Unfortunately, more than 30 million Americans suffer from an eating disorder at some point during their lives, with two-thirds of this group composed of women (Wade, et al., 2011).

Mass media is often cited as a culprit behind the growing trend of body dissatisfaction among young women (Dittmar, 2009). Mass media tends to portray women of below average thinness, and retouches and airbrushes their subjects until they reach unrealistically perfect levels of physical beauty (Hass, et al., 2012). Young women might develop body image issues if they compare themselves to social norms of beauty as displayed in these media representations (Bergstrom, et al., 2009). Indeed, the viewing of idealistically thin physical media models has been found to correlate with negative body image.

As previously mentioned, the desire for women to obtain social norms of beauty is not new, and has occurred in many forms of mass media. However, social media has created new platforms for women to develop, display, and reflect on their body image. Whereas traditional broadcast media portrays unknown professional models, social media allows users to portray themselves while viewing other "real people" that they often know in the off-line world. In this way, social media raises new questions in regard to body image.

\section{Social media}

Social media can be distinguished from more traditional broadcast media in several ways. Perloff (2014) summarizes that social media is more interactive than traditional broadcast media, and therefore provides users with more agency to personalize and control their experience. Users can select the social media platforms and expressive tools that best fit their own needs and personality. Along with this sense of personalization, social media possesses a more identity-based and relationally motivated nature than traditional broadcast media because it encourages users to display their unique selves and interact with other users on a personal level (Acquisti and Gross, 2006). As such, social media has become a vital tool for individuals to build and maintain their off-line and online reputations and relationships (Madden and Smith, 2010).

The fact that social media possesses components of identity display and relational communication indicates that these venues may have differing audience effects from traditional media. Countless studies reveal that mass media consumption has the potential to influence various dimensions of users' body image (for review, see Levine and Harrison, 2009, 2004). The connection between body image and social media, however, remains less clear.

Clues might be gathered from related research regarding the connection between social media and self-esteem. For example, Vogel, et al. (2014) found that the frequency of Facebook use predicted lower self-esteem among young adults, yet this relationship was mediated by users degree of upward social comparison. In other words, participants' self-esteem decreased as they spent more time on Facebook comparing themselves to figures of seemingly higher social status. A similar study revealed a negative correlation between self-esteem and the frequency and length of young adults' Facebook sessions (Mehdizadeh, 2010). These findings are pertinent to the present study's focus on body image due to the established link between body image and self-esteem (e.g., Fabian and Thompson, 1989). These studies, however, focused mainly on the frequency of Facebook use, and did not directly examine issues of body image or body dissatisfaction.

Facebook is a powerful and popular social media tool that incorporates text, pictures, group events, and other affordances to create an all-encompassing social media experience. Instagram, on the other hand, specifically focuses on the dissemination and consumption of personal photographs. In this way, Instagram could potentially display a more direct relationship between 
women's body image and activity on the site. Instagram selfies are a particularly interesting phenomenon, as they allow the uploading of self-taken photographs that focus on users' physical appearance. Selfies therefore provide a unique opportunity to identify correlations between self-created image content and the perception of one's body image.

\section{This study}

The image-based SNS Instagram began in 2010 and quickly rose to support more than 500 million active monthly users who shared 95 million photos every day as of June 2016 (Instagram, n.d.). The popular SNS was sold to Facebook for one billion dollars during 2012, and can now be linked with users' Facebook pages. The high social value and "buzz" surrounding Instagram makes the site a worthy topic of inquiry. The mobile aspect of Instagram provides an online platform for people to share pictures quickly and smoothly wherever they are. Simply put, it is "a smartphone application that purports to offer a new mode of instantaneous visual communication" (Champion, 2012). It encourages further social interaction through tags, comments, Likes and private messages from one user to another.

Social media has developed its own set of interactional norms. Facebook users, for example, have been found to adhere to tacit norms of friendship behavior (Bryant and Marmo, 2012). Meanwhile, Instagram has developed its own culture and etiquette (Orlander, 2014). People know what to share, what to like, as well as when and how to do these activities in ways that adhere to the latest trends. Two popular Instagram trends are \#TBT (i.e., Throwback Thursday, re-posting old pictures on Thursdays with hopes of reminiscing about the past) and \#SelfieSunday (i.e., posting self-portraits on Sundays). Although \#ThrowbackThursday remains popular, the use of selfies has spread far beyond the constraints of a single day.

Selfies have attracted a great deal of attention and curiosity because of what they can achieve in today's world. As actor James Franco (2013) described in a New York Times article, "In a visual culture, the selfie quickly and easily shows, not tells, how you're feeling, where you are, what you're doing. [...] In our age of social media, the selfie is the new way to look someone right in the eye and say, 'Hello, this is me'." [2]. In this way, the popularity of selfies might derive from their immense power as devices for displaying identity. Other users can show approval or disapproval by Liking or commenting on a selfie that has been posted; creating a media stage for attention-seeking users to put on their best identity performances and receive feedback from their peers.

Although social media has become a prevalent area of study, Instagram has received relatively little scholarly attention. Existing research has looked at Instagram's impact on human rights activism such as the ALS Ice Bucket Challenge (Herman, 2014), as well as how the site affects the dissemination and aesthetic composition of contemporary photography (Champion, 2012). The present study will extend the focus of scholarly Instagram research to the topic of selfies and body image.

Selfies have become a popular topic of media discussion. One Time article, for instance, inferred that selfies are often posted as an act of narcissism or desire of self-affirmation (Bennett, 2014). At the same time, the article argued that selfies could provide women the power to redefine beauty and challenge the thin ideal that has become the media norm. The potential for selfies to subvert unhealthy body ideals might, however, remain unrealized if users continue to uphold current social norms in their use of selfies.

The present study seeks to better understand the phenomenon of Instagram selfies, particularly in relation to the body image of young adult women. Before examining the relationship between selfies and body image, it is first necessary to determine the prevalence of selfies among young adult women. To do so, the present study will distinguish between how many selfies the average young woman: 1) takes, and 2) posts to Instagram. This distinction enables potentially divergent trends. For example, some women might take a large number of selfies for their own purposes, yet post only a small number of these selfies for others to see. Moreover, taking selfies can be conceptualized as a private activity, whereas posting selfies takes on an inherently public nature that involves a desire, or at the very least willingness, to garner attention from others. This distinction could be of importance when considering the relationship between selfies and body image. As a result, the following question will be tested:

\section{$R Q 1:$ How frequently do female Instagram users 1) take selfies, and 2) post selfies?}

As previously mentioned, there is much debate regarding the effects that social media posts such as selfies might have on young women, specifically in regard to body image. Body image can be assessed in many different ways, but the present study will focus on body dissatisfaction by asking participants to consider their own body image in relation to their unique sense of the ideally beautiful body.

The present study also seeks to understand whether actual body size is related to participants' likelihood of taking and posting selfies, which will be accomplished by calculating each participant's body mass index (BMI). Hence, actual body size represents a more objective indicator of participant's bodies, which is not sensitive to personal perception. That said, it is likely that these two body-related indicators will be related, such that participants with a higher BMI will report greater body dissatisfaction. The following hypothesis will be tested:

\section{H1: Participants actual body size (i.e., BMI) will be positively related to their reported sense of body dissatisfaction.}

Various predictions might be made regarding the potential associations between actual body size, body dissatisfaction, and selfies. It is possible that young womens' use of selfies is partially informed by their actual body size. At the same time, it is plausible that actual body size matters much less than a participant's sense of (dis)satisfaction with her size. A woman who is content with a larger body size, for example, might be more likely to proudly share a selfie than a relatively smaller woman who suffers from poor body image. As previously reviewed, selfies might be seen as an act of identity display as well as an attention-seeking behaviour. In either case, the taking and/or posting of selfies might be connected with one's sense of body 
image. Whether this relationship is positive or negative, however, is more debatable. On one hand, young women who experience a large amount of body dissatisfaction might take and/or post selfies in an attempt to garner affirming messages that might boost their own self-confidence. In this case, body dissatisfaction might positively correlate with the number of selfies taken and posted. On the other hand, individuals with poor body image might be too self-conscious to take and/or post selfies that would draw attention to their bodies; implying a potential negative relationship between body dissatisfaction and the taking and/or posting of selfies. Due to these divergent speculations, the present study will test the following research questions:

$R Q 2:$ Does actual body size predict the number of selfies taken?

$R Q 3:$ Does body dissatisfaction predict the number of selfies taken?

$R Q 4:$ Does actual body size predict the number of selfies posted to Instagram?

$R Q 5$ : Does body dissatisfaction predict the number of selfies posted to Instagram?

\section{Methods}

\section{Participants and procedures}

The sample consisted of 130 female college students ranging from $18-32$ years of age $(M=19.9 ; S D=1.62)$. Of the sample, 28 percent were freshmen, 57 percent were sophomores, nine percent were juniors, and six percent were seniors. This sample size was appropriate because analysis using $\mathrm{G}^{*}$ Power revealed that a sample of 107 participants would provide enough power to detect moderate effect sizes for the statistical tests conducted within the present study.

Participants were recruited via Facebook to complete an online survey. After posting the survey link to their own pages and various university and group pages, the researchers created a snowball effect by asking other users to share the link. After providing informed consent, participants completed a three-part questionnaire. After confirming their status as active Instagram users, the first portion of the survey asked participants to answer a series of questions about their selfie behavior. Using a definition from Storella (2014), participants were provided with the following instructions:

Answer the following based on your personal Instagram account. If you cannot recall specifics, scroll through your Instagram feed. Please keep in mind the definition of a selfie, which is "a digital self-portrait usually taken with the use of a camera phone."

To further increase clarity, participants were asked to focus only on solo-selfies (i.e., self-portraits containing only their own image) rather than group-selfies (i.e., self-portraits taken by one member of a group; also known as "group hold-outs" or "groupie" photos). The second part of the questionnaire included items about participants' physical bodies and body image. The final portion of the survey collected basic demographic information. All study procedures were given approval by the Institutional Review Board at the researchers' university.

\section{Measures}

Number of selfies taken and posted. In attempting to assess selfie-related behavior, it was necessary to determine an adequate length of time that was long enough to provide variability, yet short enough to enable participant recall. An article from the Telegraph estimated that the "average person posts over 100 selfies a year." [3]. This number of selfies breaks down to less than 10 per month, which was determined to be a small enough number for most individuals to comprehend with relative accuracy. Hence, the number of selfies taken was assessed using an open-ended item that asked participants how many solo-selfies they take - in general - during a typical month. Likewise, the number of selfies posted was similarly measured using an open-ended question that asked participants how many solo-selfies they post to Instagram during a typical month.

Actual body size. Participants' actual body size was assessed in the form of their body mass index (BMI). The BMI is a commonly used indicator of body size that is calculated by measuring a person"s weight (in kilograms) and dividing it by their height squared (in centimeters). According to the National Institute of Health (n.d.), BMI ranges are commonly defined using the following ranges: less than 18.5 is underweight; $18.5-24.9$ is normal weight; $25-29.9$ is overweight; and greater than 30 is obese. The average BMI in the present study was $21.59(S D=3.17)$.

Body dissatisfaction. Body dissatisfaction was assessed using the Body Image Assessment Scale-Body Dimensions (or BIASBD). The BIAS-BD, tested in a study by Gardner, et al. (2009), uses a pictorial scale of silhouette figures that are designed to represent various body sizes that range from 60-140 percent of the typical female BMI. The BIAS-BD was originally created by the U.S. Air Force Material Command using a program known as GEBOD, or the Generator of Body Data. The GEBOD drew from a sample of 4,325 participants, and utilized a regression formula to create silhouettes at five percent intervals of body size from 60 percent to 140 percent of the average female body; each of the 17 silhouettes represents a different BMI while maintaining the same body proportions.

Participants in the present study were provided with the BIAS-BD's pictorial scale of silhouette figures (See Figure 1), and asked to answer the following questions: 1) "Of the body silhouettes shown above, indicate the one that most accurately resembles your current body size," and 2) "Of the body silhouettes shown above, indicate the one that best represents your ideal body size." Response options ranged from 1-17, with 1 representing the smallest silhouette at 60 percent of the typical 
adult female BMI, and 17 representing the largest silhouette representing a person with a BMI of 140 percent of the typical adult female BMI. Body dissatisfaction was assessed by calculating the difference between the BMI percentage associated with the selected current body size silhouette, and BMI percentage corresponding with the selected ideal body size silhouette. Absolute values were utilized so that it did not matter how participants were dissatisfied (i.e., as too small or too large); only the extent to which they wanted a different size than their current perceived size. As such, body dissatisfaction scores were represented in the form of a percentage, with 0 percent representing no desired body change, and subsequently larger scores representing proportionately larger desired body change. On average, participants indicated that that their current body was best represented by the silhouettes corresponding to 85 percent of the typical female body size, and that their ideal body size would correspond with the silhouette representing 75 percent of the normal female body size.

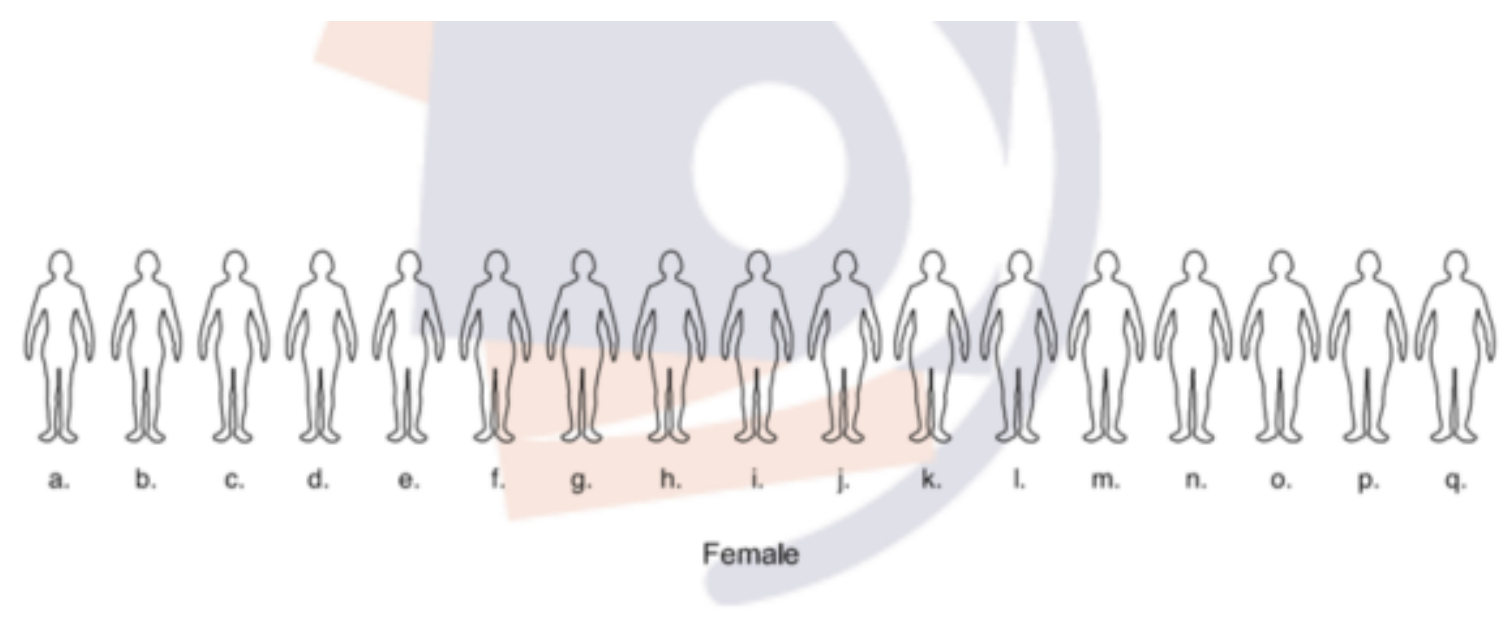

Figure 1: BIAS-BD scale item.

\section{Results}

$R Q 1$ asked how frequently female Instagram users take selfies and post them to Instagram. Results indicated that participants were far more likely to take selfies than they were to actually post them. Participants reported taking an average of approximately 17 selfies each month $(S D=41.836)$, yet averaged only 0.34 selfies uploaded to Instagram during an average month $(S D=.83)$.

$H 1$ predicted that actual body size (BMI) would be positively related to participants' sense of body dissatisfaction. Results from a bivariate correlation test revealed a moderate positive correlation between actual body size $(M=21.59, S D=3.17)$ and body dissatisfaction $(M=13.76, S D=11.74) ; r=.44, p<.001$. Actual body size accounted for 19.4 percent of the variance in body dissatisfaction. As such, $H 1$ was supported.

$R Q 2$ asked whether a woman's actual body size would be related to the number of selfies taken. $R Q 3$ similarly asked whether a significant relationship would exist between a woman's sense of body dissatisfaction and the number of selfies taken. These two research questions were tested using linear regression analysis with selfies taken per month serving as the criterion variable, and actual body size and perceived body dissatisfaction serving as the predictor variables. Age was considered as a potential covariate, but was removed due to a lack of significance within the final model. The final model revealed a significant multivariate effect on the number of selfies taken per month: $R=.29$, Total $R^{2}=0.09$; adjusted $R^{2}=0.07 ; F(2,103)=4.76, p$ $=.01$. Collinearity diagnostics were unremarkable $(\mathrm{VIF}=1.23$, Tolerance $=.81)$. More specifically, actual body size $(B=-2.91$, $\mathrm{SE} B=1.38, \beta=-.22, p=.04)$ and perceived body dissatisfaction $(B=1.10, \mathrm{SE} B=.37, \beta=.31, p=.004)$ both predicted the number of selfies taken per month. In response to $R Q 2$, actual body size was inversely related to the number of selfies taken; such that possessing a lower BMI predicted more selfies taken. In response to $R Q 3$, results revealed a positive relationship between body dissatisfaction and selfies taken; such that being more dissatisfied with one's body predicted more selfies taken. Of note, body dissatisfaction displayed larger weighting within the overall model.

$R Q 4$ asked whether participants' actual BMI would predict the number of selfies they upload to Instagram. RQ5 similarly explored whether participants' sense of body dissatisfaction would predict the number of selfies they upload to Instagram. These questions were tested using linear regression analysis with the number of selfies uploaded to Instagram as the criterion variable, and participant's actual body size (BMI) and perceived body dissatisfaction serving as the predictors. Once again, participant age was considered yet ultimately removed as a covariate due to a lack of significance. The final model did not reveal a significant multivariate effect on the number of selfies posted to Instagram per month: $R=.10$, Total $R^{2}=0.011$; 
adjusted $R^{2}=-.009 ; F(2,104)=.54, p=.58$. More specifically, neither actual body size $(\mathrm{B}=-.03, \mathrm{SE} \mathrm{B}=.03, \beta=-.10, p=$ .37) nor body dissatisfaction ( $\mathrm{B}=-.001$, $\mathrm{SE} B=.008, \beta=-.007, p=.95)$ reached univariate significance. In response to the research questions, actual body size (RQ4) and body dissatisfaction (RQ5) were not related to the number of selfies uploaded to Instagram per month.

\section{Discussion}

The present study examined the connections between selfies, actual body size (BMI), and body dissatisfaction. This set of variables enabled two important distinctions that contribute to the growing body of literature regarding body image and social media: 1) between the effects of actual body size and body image perception, and 2) between selfies taken and selfies posted to Instagram. Several important take-away points can be gleaned from the present study's results.

\section{Body image and actual body size}

Analysis of $\mathrm{H} 1$ revealed a moderate relationship between the actual body size and body dissatisfaction, which offers two interesting pieces of information. First, although the variables were related, this relationship accounted for only 20 percent of the total variance. This implies that actual body size and body dissatisfaction should, indeed, be considered separately. Second, the moderate positive relationship signals that despite other potential factors of importance, individuals with a larger body size are more likely to report body dissatisfaction. This finding is fairly intuitive - overweight or obese individuals will likely experience more body dissatisfaction - yet the implications are actually somewhat more complex when the specific results are interrogated.

Although not directly related to a hypothesis or research question, the reported statistics related to actual body size (BMI) and body dissatisfaction (based on the BIAS-BD) speak toward the state of body image perception for women in America. According to the National Institutes of Health (n.d.), the ideal BMI range for a healthy adult falls between 18.4 and 24.9. The average $\mathrm{BMI}$ in the present study was $21.59(S D=3.17)$; indicating that the majority of participants were within a perfectly healthy BMI range. That said, the average participant's score for body dissatisfaction revealed a desire for an approximately 10 percent change in body size, and this change was almost exclusively oriented towards being more thin. This finding supports previous claims (e.g., Bergstrom, et al., 2009), suggesting that the thin ideal portrayed in media has affected the way that young women view their own bodies.

\section{Predicting the frequency of selfies taken and posted}

The present study distinguished between the number of solo selfies taken per month and the number of solo selfies posted to Instagram per month. This distinction was made to better understand whether the more private act of taking selfies diverges from the inherently public act of posting selfies on Instagram. Results from $R Q 1$ indicated that young women are far more likely to take selfies than they are to post them. The average woman reported posting less than one selfie to Instagram during an average month, despite taking an average of 17 selfies during the same time period. Although many people are quick to assume that selfies are motivated by a narcissistic need for attention, these results suggest that selfies likely serve a more private and internal purpose for young women. The present study did not seek to examine user's motives for taking and posting selfies, but doing so could provide a useful line of future research.

Our findings for $R Q 2-R Q 5$ revealed that the decision to distinguish between selfies taken and selfies posted to Instagram was extremely important. Actual body size and perceived body dissatisfaction both predicted the number of selfies taken (with body dissatisfaction as the strongest predictor), yet neither significantly predicted the number of selfies uploaded to Instagram. One statistical explanation for this finding is that there was more variability in the number of selfies taken than in the number of selfies posted. Another potential explanation lies within the private/public nature of the two types of selfie activity. Taking selfies is private and therefore likely to be driven by internally focused motivations, whereas posting selfies is likely to involve aspects of external attention and validation. When interpreted together, these results suggest that women of divergent sizes and levels of body satisfaction are equally likely to post selfies in the public Instagram venue, yet might differ in the internal processes that drive their decisions to take selfies. While it is semi-perplexing that being both thin and dissatisfied with one's body predicted selfie taking in the present study, these results suggest that there are additional variables at work. For example, future research should examine the gratifications that young women obtain from both privately taking and publicly posting selfies to determine whether these gratifications are related to body size and body image. Perhaps some women take selfies to celebrate their bodies, while others take selfies to monitor and/or judge their bodies. Likewise, future research should explore whether women with divergent body sizes and body images are likely to take different kinds of selfies; such as body centric versus facial centric images. It is plausible that body size and body image influence the actual qualitative nature of the selfie photos that young women take and post. These possibilities are speculative, however, as they extend beyond the reach of the present study.

\section{Limitations and conclusions}

The present study provides an exploratory analysis of how body size and body image influences the taking and posting of selfies. This analysis provides much insight, but is not without limitations. First, the present study possessed enough power to detect results, but was still limited by the relatively small and convenience-based nature of the sample. Future research 
should correct this by expanding to other populations (e.g., male Instagram users and different age groups). Given that norms of body beauty are culturally situated (Szymanski, et al., 2011), it would also be useful for researchers to test similar concepts using an international sample.

A second limitation is that the present study focused purely on Instagram, and did not look at the posting of selfies on other forms of social media. Young women likely select from an array of social media tools such as Facebook, Instagram, and Snapchat when posting different types of content. Future research should seek to determine whether the affordances of each platform might provoke differences in selfie-related behaviors. Likewise, the present study examined the number of selfies posted on Instagram, but did not assess the overall amount of time spent using Instagram or other forms of social media. The overall time spent on Instagram and other social media sties - even if passively browsing content - could be an important variable for future research to examine in relation to body image perception.

A third limitation is that the present study did not assess potentially relevant psychological variables such as self-esteem and social comparison. Existing research suggests that self-esteem is related to body image (Fabian and Thompson, 1989), and that both self-esteem and upward social comparisons are associated with the frequency of Facebook use (Mehdizadeh, 2010; Vogel, et al., 2014). Future research should therefore seek to address the role of self-esteem and social comparison within the context of body image perception, selfies, and social media.

A fourth limitation lies within the present study's use of BMI as an indicator of actual body size. As previously discussed, BMI is calculated by measuring a person's weight (in kilograms) and dividing it by their height squared (in centimeters) (National Institutes of Health, n.d.). BMI can be easily calculated based on information that is readily available to most participants, and is therefore an ideal indicator of actual body size for naturalistic social science research. BMI cannot, however, be interpreted as indicative of overall body health because it does not account for a person's percentage of lean and fatty body mass. This limitation is important because current cultural norms of body beauty tend to privilege thinness (Pelegrini, et al., 2014). Recent social movements, such as the fat acceptance movement, have sought to disrupt the anti-fat discourse that can provoke forms of body-based shaming and discrimination (see Cooper, 2010). The medical warrants of this movement are widely debated, but its popularity indicates that more and more women are seeking ways to reaffirm their own bodies while rejecting the pressure to be thin. Future research should therefore seek to examine selfie taking and posting within the context of not only BMI, but also: 1) participants' actual body composition (e.g., body fat percentage), and 2) participants' perceptions regarding the desirability of thinness and/or acceptance of fatness.

Along similar lines, the present study was able to discern that women who are more dissatisfied with their bodies tend to take mores selfies per month. This could be attributed to the desire of women with negative body image perception to find just the right angle or lighting that will contribute to the ideal selfie. However, more research would be required to confirm any explanation for our results. Such research should focus on the relationship of social media usage with selfies and body image perception, as well as how self-esteem relates to body image perception in the setting of social media.

Regardless of these limitations, the present study takes significant steps toward determining how actual body size and body image perception relates to frequency of selfies taken and posted by young adult women. As social media and selfies become more prevalent in today's modern media culture, body image perception may become more and more at risk of distortion. The present study serves as a springboard for additional research regarding various ways that body image distortion might be prevented and confronted. FM

\section{About the authors}

Charles Wagner graduated from Trinity University in 2016 with a Business Analytics and Technology and Communication double major. He is currently following his passions for helping others and solving complex problems by working for PriceWaterhouseCoopers as a technology consultant in Chicago. He hopes one day to return to school for his MBA and continue his journey to learn, practice, and teach all things technology and communication.

Ester Aguirre is a current student at Trinity University in San Antonio, Texas. She will graduate with a degree in Political Sciences and a minor in Spanish.

Erin M. Sumner (Ph.D. 2012, Arizona State University) is an Assistant Professor of Human Communication at Trinity University in San Antonio, Texas. Her primary research interests examine the interpersonal and relational dynamics of computer-mediated communication. Her work has appeared in journals such as Communication Monographs, Journal of Computer-Mediated Communication, New Media \& Society, Journal of Social and Personal Relationships, Communication Methods and Measures, and Communication Reports.

E-mail: ebryant [at] trinity [dot] edu

\section{Notes}

1. See http://blog.oxforddictionaries.com/press-releases/oxford-dictionaries-word-of-the-year-2013/, accessed 16 August 2016.

2. Franco, 2013, paragraphs 10-13.

3. Sparkes, 2015, p. 1. 


\section{References}

A. Acquisti and R. Gross, 2006. "Imagined communities: Awareness, information sharing, and privacy on the Facebook," In: P. Golle and G. Danezis (editors). Privacy enhancing technologies. Lecture Notes in Computer Science, volume 4258. Berlin: Springer, pp. 36-58. doi: http://dx.doi.org/10.1007/11957454 3, accessed 16 August 2016.

J. Bennett, 2014. "Our bodies, our selfies: The feminist photo revolution," Time (11 August), at http://www.time.com/3099103/feminist-selfies-uglyfeminists-iwokeuplikedis/, accessed 16 August 2016.

R.L. Bergstrom, C. Neighbors, and J.E. Malheim, 2009. "Media comparisons and threat to body image: Seeking evidence of self-affirmation," Journal of Social and Clinical Psychology, volume 28, number 2, pp. 264-280. doi: http://dx.doi.org/10.1521/jscp.2009.28.2.264, accessed 16 August 2016.

d. boyd and N.B. Ellison, 2007. "Social network sites: Definition, history, and scholarship," Journal of Computer-Mediated Communication, volume 13, number 1, pp. 210-230. doi: http://dx.doi.org/10.1111/j.1083-6101.2007.00393.x, accessed 16 August 2016.

E.M. Bryant and J. Marmo, 2012. "The rules of Facebook friendship: A two-stage examination of interaction rules in close, casual, and acquaintance friendships," Journal of Social and Personal Relationships, volume 29, number 8, pp. 1,013-1,035. doi: http://dx.doi.org/10.1177/0265407512443616, accessed 16 August 2016.

T.F. Cash, 2002. "Cognitive behavioral perspectives on body image," In: T F. Cash and T. Pruzinsky (editors). Body image: $A$ handbook of theory, research, and clinical practice. New York: Guilford Press, pp. 38-46.

C. Champion, 2012. "Instagram: Je-suis-là?" Philosophy Of Photography, volume 3, number 1, pp. 83-88. doi: http://dx.doi.org/10.1386/pop.3.1.83 7, accessed 16 August 2016.

C. Cooper, 2010. "Fat studies: Mapping the field," Sociology Compass, volume 4, number 12, pp. 1,020-1,034. doi: http://dx.doi.org/10.1111/j.1751-9020.2010.00336.x, accessed 16 August 2016.

T.E> Davison and M.P McCabe, 2005. "Relationships between men's and women's body image and their psychological, social, and sexual functioning," Sex Roles, volume 52, number 7, pp. 463-475. doi: http://dx.doi.org/10.1007/s11199-005-3712-z, accessed 16 August 2016.

H. Dittmar, 2009. "How do 'body perfect' ideals in the media have a negative impact on body image and behaviors? Factors and processes related to self and identity," Journal of Social and Clinical Psychology, volume 28, pp. 1-8. doi: http://dx.doi.org/10.1521/jscp.2009.28.1.1, accessed 16 August 2016.

L.J. Fabian and J.K. Thompson, 1989. "Body image and eating disturbance in young females," International Journal of Eating Disorders, volume 8, number 1, pp. 63-74. doi: http://dx.doi.org/10.1002/1098-108X(198901)8:1<63::AID-EAT2260080107>3.0.CO;2-9, accessed 16 August 2016.

J. Franco, 2013. "The meanings of the selfie," New York Times (26 December), at http://www.nytimes.com/2013/12/29/arts/the-meanings-of-the-selfie.html, accessed 16 August 2016.

B.L. Fredrickson and T.-A. Roberts, 1997. "Objectification theory: Toward understanding women's lived experiences and mental health risks," Psychology of Women Quarterly, volume 21, number 2, pp. 173-206. doi: http://dx.doi.org/10.1111/i.1471-6402.1997.tb00108.x, accessed 16 August 2016.

R. Fry, 2016. "Millennials overtake baby boomers as America's largest generation," Pew Research Center (25 April), at http://www.pewresearch.org/fact-tank/2016/04/25/millennials-overtake-baby-boomers/, accessed 16 August 2016.

A. Furnham, N. Badmin, and I. Sneade, 2002. "Body image dissatisfaction: Gender differences in eating attitudes, selfesteem, and reasons for exercise," Journal of Psychology, volume 136, number 6, pp. 581-596. doi: http://dx.doi.org/10.1080/00223980209604820, accessed 16 August 2016.

R.L. Gardner, L.M. Jappe, and L. Gardner, 2009. "Development and validation of a new figural drawing scale for body-image assessment: The BIAS-BD," Journal of Clinical Psychology, volume 65, number 1, pp. 113-122. doi: http://dx.doi.org/10.1002/iclp.20526, accessed 16 August 2016.

A.L. Gonzales and J.T. Hancock, 2011. "Mirror, mirror on my wall: Effects of exposure to Facebook on self-esteem," Cyberpsychology, Behavior and Social Networking, volume 14, numbers 1-2, pp. 79-83. doi: http://dx.doi.org/10.1089/cyber.2009.0411, accessed 16 August 2016.

C.J. Hass, L.A. Pawlow, J. Pettibone, and D.J. Segrist, 2012. "An intervention for the negative influence of media on body esteem," College Student Journal, volume 46, number 2, pp. 405-418.

J. Herman, 2014. "Hashtags and human rights: Activism in the age of Twitter," Carnegie Council for Ethics in International Affairs (12 November), at doi: http://www.carnegiecouncil.org/publications/ethics online/0099, accessed 16 August 2016. 
N. Howe and N. Strauss, 2000. Millennials rising: The next great generation. New York: Vintage.

Instagram.com, n.d. "Press news," at https://www.instagram.com/press/, accessed 16 August 2016.

M.P. Levine and K. Harrison, 2009. "Effects of media on eating disorders and body image," In: J. Bryant and M.B. Oliver (editors). Media effects: Advances in theory and research Third edition. New York: Routledge, pp. 490-516.

M.P. Levine and K. Harrison, 2004. "Media's role in the perpetuation and prevention of negative body image and disordered eating," In: J.K. Thompson (editor). Handbook of eating disorders and obesity. Hoboken, N.J.: Wiley, pp. 695-717.

S.M. Livingstone, 2002. Young people and new media: Childhood and the changing media environment. Thousand Oaks, Calif.: Sage.

M. Madden and A. Smith, 2010. "Reputation management and social media," Pew Research Center (26 May), at http://pewinternet.org/Reports/2010/Reputation-Management, accessed 16 August 2016.

S. Mehdizadeh, 2010. "Self-presentation 2.0: Narcissism and self-esteem on Facebook," Cyberpsychology, Behavior and Social Networking, volume 13, number 4, pp. 357-364.

doi: http://dx.doi.org/10.1089/cyber.2009.0257, accessed 16 August 2016.

National Institutes of Health, n.d. "Calculate your body mass index," at

http://www.nhlbi.nih.gov/health/educational/lose wt/BMl/bmicalc.htm, accessed 16 August 2016.

R. Olivardia, H.G. Pope, J.J. Borowiecki, and G.H. Cohane, 2004. "Biceps and body image: The relationship between muscularity and self-esteem, depression, and eating disorder symptoms," Psychology of Men \& Masculinity, volume 5, number 2, pp. 112-120.

doi: http://dx.doi.org/10.1037/1524-9220.5.2.112, accessed 16 August 2016.

S. Orlander, 2014. "4 trends on Instagram exposed," Huffington Post (9 January), at http://www.huffingtonpost.com/uloop/4trends-on-instagram-exp_b_4565076.html, accessed 16 August 2016.

A. Pelegrini, C. Sacomori, M.C. Santos, F.F. Sperandio, and F.L. Cardoso, 2014. "Body image perception in women: Prevalence and association with anthropometric indicators," Brazilian Journal of Kineanthropometry \& Human Performance, volume 16, number 1, pp. 58-65.

doi: http://dx.doi.org/10.5007/1980-0037.2014v16n1p58, accessed 16 August 2016.

R. Perloff, 2014. "Social media effects on young women's body image concerns: Theoretical perspectives and an agenda for research," Sex Roles, volume 71, number 11, pp. 363-377.

doi: http://dx.doi.org/10.1007/s11199-014-0384-6, accessed 16 August 2016.

J.A. Pesa, T.R. Syre, and E. Jones, 2000. "Psychosocial differences associated with body weight among female adolescents: The importance of body image," Journal of Adolescent Health, volume 26, number 5, pp. 330-337.

doi: http://dx.doi.org/10.1016/S1054-139X(99)00118-4, accessed 16 August 2016.

M. Sparkes, 2015. "The 'selfie' craze: Who are more vain, men or women?" Telegraph (29 January), at http://www.telegraph.co.uk/technology/11376259/The-selfie-craze-who-are-more-vain-men-or-women.html, accessed 16 August 2016.

A.C. Storella, 2014. "It's selfie-evident: Spectrums of alienability and copyrighted content on social media," Boston University Law Review, volume 94, pp. 2,045-2,088, and at http://www.bu.edu/bulawreview/files/2014/12/STORELLA.pdf, accessed 16 August 2016.

W. Strauss and N. Howe, 1991. Generations: The history of America's future, 1584 to 2069. New York: Morrow.

D.M. Szymanski, L.B. Moffitt, and E.R. Carr, 2011. "Sexual objectification of women: Advances to theory and research," Counseling Psychologist, volume 39, number 1, pp. 6-38.

doi: http://dx.doi.org/10.1177/0011000010378402, accessed 16 August 2016.

E.A. Vogel, J.P. Rose, L.R. Roberts, and K. Eckles, 2014. "Social comparison, social media, and self-esteem," Psychology of Popular Media Culture, volume 3, number 4, pp. 206-222.

doi: http://dx.doi.org/10.1037/ppm0000047, accessed 16 August 2016.

T.D. Wade, A. Keski-Rahkonen, and J. Hudson 2011. "Epidemiology of eating disorders," In: M. Tsuang, M. Tohen, and P.B. Jones (editors). Textbook in psychiatric epidemiology. Third edition. Hoboken, N.J.: Wiley, pp. 343-360.br>doi: http://dx.doi.org/10.1002/9780470976739.ch20, accessed 16 August 2016.

J. White and E. Halliwell, 2010. "Examination of a sociocultural model of excessive exercise among male and female adolescents," Body Image, volume 7, number 3, p. 227-233.

doi: http://dx.doi.org/10.1016/j.bodyim.2010.02.002, accessed 16 August 2016.

\section{Editorial history}


Received 18 January 2016; revised 1 August 2016; accepted 16 August 2016.

\section{(c) (i) (2) (-)}

This paper is licensed under a Creative Commons Attribution-NonCommercial-ShareAlike 4.0 International License.

The relationship between Instagram selfies and body image in young adult women

by Charles Wagner, Ester Aguirre, and Erin M. Sumner.

First Monday, Volume 21, Number 9 - 5 September 2016

http://firstmonday.org/ojs/index.php/fm/rt/printerFriendly/6390/5620

doi: http://dx.doi.org/10.5210/fm.v21i9.6390 